\title{
Nosocomial Infections in Gastrointestinal Cancer Patients: Bacterial Profile, Antibiotic Resistance Pattern, and Prognostic Factors
}

This article was published in the following Dove Press journal: Cancer Management and Research

\author{
Ai-Min Jiang' \\ Na Liu iD ' \\ Rim Ali Said ${ }^{2}$ \\ Meng-Di Ren' \\ Huan Gao' \\ Xiao-Qiang Zheng (iD) \\ Xiao Fu' \\ Xuan Liang' \\ Zhi-Ping Ruan' \\ Yu Yao' \\ Tao Tian iD ${ }^{\prime}$ \\ 'Department of Medical Oncology, The \\ First Affiliated Hospital of Xi'an Jiaotong \\ University, Xi'an, Shaanxi 7I006I, \\ People's Republic of China; ${ }^{2}$ Department \\ of Imaging and Nuclear Medicine, The \\ First Affiliated Hospital of Xi'an Jiaotong \\ University, Xi'an, Shaanxi 71006I, \\ People's Republic of China
}

Correspondence: Tao Tian; Yu Yao Department of Medical Oncology, The First Affiliated Hospital of Xi'an Jiaotong University, No. 277 Yanta West Road, Xi'an, Shaanxi 71006I, People's Republic of China

Tel +86-29-85324600

Fax +86-29-85324086

Email tiantao0607@163.com;

1357210161I@163.com
Background: Cancers of the gastrointestinal (GI) tract and its associated excretory glands are one of the most common causes of cancer-related death worldwide, and these patients are more likely to developing nosocomial infections due to immunodeficiency.

Objective: To explore the bacterial profile, antibiotic resistance pattern, and prognostic factors of nosocomial infections in hospitalized GI cancer patients.

Methods: All electronic medical records of nosocomial infection episodes in hospitalized GI cancer patients were retrospectively reviewed. In-hospital mortality was used to evaluate the prognosis of patients. Mann-Whitney test, Chi-square test, and binary logistic regression analysis were used to identify potential risk factors for in-hospital mortality. $P$-values $<0.05$ were considered statistically significant.

Results: A total of 428 GI cancer patients developed nosocomial infections during hospitalization. Respiratory tract infections $(44.2 \%)$, bloodstream infections (BSIs) $(11.7 \%)$, and abdominal cavity infections (11.4\%) were the most common infection sites. The predominant causative pathogens were extended-spectrum $\beta$-lactamase (ESBL)-producing Escherichia coli (13.6\%), ESBL-negative E. coli (11.9\%), and Klebsiella pneumoniae (10.0\%). Multidrug-resistant (MDR) strains were detected in $27.6 \%$ of isolates. Antimicrobial susceptibility analysis showed that the isolated Gram-negative bacteria (GNB) exhibited high sensitivity to amikacin, meropenem, imipenem, and piperacillin/tazobactam, while the isolated Gram-positive bacteria exhibited high sensitivity to tigecycline, linezolid, and vancomycin. The overall in-hospital mortality of all patients was $11.2 \%$ in the study. Multivariate analysis showed that ECOG performance status $\geq$ two scores, length of antibiotic treatment $<9.0$ days, existence of septic shock, and hypoproteinemia were independent risk factors for in-hospital mortality.

Conclusion: The burden of nosocomial infections in GI cancer patients is considerably high, with GNB being predominantly isolated causative pathogens. Surveillance on serum albumin level, adequate antibiotic treatment, early identification, and prompt treatment of septic shock could benefit the prognosis.

Keywords: nosocomial infections, gastrointestinal cancer, bacterial profile, antibiotic resistance, prognostic factors

\section{Introduction}

Cancers of the gastrointestinal (GI) tract and its associated excretory glands, also known as digestive cancer, are one of the most common causes of cancer-related death worldwide, and it remains a major public health concern for past decades. ${ }^{1}$ Although surgery, chemotherapy, radiotherapy, molecular targeted therapy, and 
immunotherapy have significantly improved the survival of GI cancer patients in recent years, it remains a bleak prognosis. Furthermore, these patients are predisposed to developing nosocomial infections due to immunodeficiency caused by malignancy itself and its treatments. ${ }^{2}$ Even though most infections are temporary, the consequences can last longer.

Nosocomial infections in cancer patients delay the initiation of chemotherapy and reduce the routine dosage. ${ }^{2,3}$ In addition, it prolongs hospitalization, raises morbidity and mortality, and increases the financial burden of patients and their families. ${ }^{3-5}$ Therefore, early identification of infection episodes and swift initiation of appropriate antibiotic treatment is pivotal for cancer patients, and it plays an important role in reducing infection-associated mortality. ${ }^{2}$ As far as we know, previously published studies have merely focused on bloodstream infections (BSIs) in cancer patients. In fact, except for BSIs, urinary tract infections, respiratory infections, and gastrointestinal tract infections are more common in nosocomial infections. ${ }^{6}$ Herein, we conducted the present study to extensively describe the microbiological distribution, antibiotic resistance pattern, and clinical outcomes of nosocomial infections in hospitalized GI cancer patients.

\section{Materials and Methods Study Design}

This retrospective observational study enrolled all hospitalized GI cancer patients with nosocomial infections at the First Affiliated Hospital of Xi'an Jiaotong University, cancer treatment center from August 2013 to June 2019. The hospital is a 2560-bed medical center in northwestern China. Surgery, chemotherapy, radiotherapy, and immunotherapy are the most common treatment for cancer patients in the cancer treatment center. The electronic medical records of all included GI cancer patients were reviewed during the study period. All cancer patients diagnosed with nosocomial infection below the age of 18 or without complete medical records were excluded. This study was conducted under the requirements of the Declaration of Helsinki.

\section{Data Collection}

All data were extracted from electronic medical records and recorded in a standardized form in Excel. Demographic and clinical data included gender, age, tobacco use, underlying cancer type, the existence of distant metastasis, disease stage, Eastern Cooperative Oncology Group (ECOG) performance status, comorbidities, the Charlson comorbidity index $(\mathrm{CCI}){ }^{7}$ the degree of fever, methods of cancer treatment (surgery, chemotherapy, radiotherapy, or concurrent chemoradiotherapy), recent corticosteroid treatment, Granulocyte colony-stimulating factor (G-CSF) usage, and the type of indwelling catheters. Infection-related data included primary sites of infection, empirical antibiotic treatment, effective antibiotic treatment, the existence of septic shock, the use of ventilator, intensive care unit (ICU) admission, length of antibiotic treatment, length of hospitalization, and clinical outcomes of the analyzed infection episodes (death or discharged during hospitalization). Laboratory examination parameters mainly included blood routine test (haemoglobin, platelet count, white-cell count, neutrophils count, and lymphocytes count), serum albumin level, serum procalcitonin (PCT) concentration, and drug susceptibility tests of the isolated pathogens.

\section{Definitions}

GI cancer patients were defined as infection episodes in accordance with the following conditions: (a) at least one positive for pathogen was found in the clinical sample during hospitalization; (b) according to the criteria described previously, ${ }^{8}$ there were corresponding symptoms and signs records, and the laboratory examination results or radiology results indicated the presence of nosocomial infection; or (c) diagnosis of infection was clearly recorded in electronic medical records. ${ }^{9}$ Polymicrobial infection was defined as two or more different pathogens were isolated from clinical samples during hospitalization.

Fever was defined as an axillary temperature above $38.3{ }^{\circ} \mathrm{C}$ or two consecutive readings of temperature was above $38{ }^{\circ} \mathrm{C} .{ }^{10}$ Shock was considered if systolic blood pressure lower than $90 \mathrm{mmHg}$, and there was no response after administration of fluid therapy and/or vasoactive medications. ${ }^{11}$

According to the drug susceptibility tests of the isolated pathogen, the empirical antibiotic treatment regimen was considered effective if it included at least one antibiotic that was in vitro active against the microorganism. ${ }^{9}$ Otherwise, it was considered ineffective. ${ }^{9}$

Multidrug-resistant (MDR) strains were classified into multidrug-resistant Gram-negative bacteria (MDRGNB) and MDR Gram-positive bacteria. The isolated pathogen was defined as MDRGNB if it fulfilled any of the conditions: (a) extended-spectrum $\beta$-lactamase (ESBL)-producing Enterobacteriaceae; (b) AmpC cephalosporinase hyperproducing Enterobacteriaceae; (c) carbapenem-resistant 
Enterobacteriaceae (CRE); (d) Stenotrophomonas maltophilia; and (e) MDR Pseudomonas aeruginosa and Acinetobacter baumannii. ${ }^{12}$ MDR Gram-positive bacteria included vancomycin-resistance Enterococcus faecium, and methicillin-resistant Staphylococcus aureus (MRSA). ${ }^{12}$

\section{Antimicrobial Susceptibility Test and PCT Measurement}

The commercial panels CN1S from the MicroScan automated system (Siemens Healthcare Diagnostics Ltd) or Vitek 2 Compact automated microbiology system (BioMerieux, Craponne, France) were adopted to identify microorganisms and drug susceptibility test. In accordance with the Clinical and Laboratory Standards Institute (CLSI) guidelines, the double-disc synergy test with EDTA discs was adopted to screen metallo- $\beta$-lactamase producers, and the modified Hodge method was applied to identify carbapenemase producers. ${ }^{13}$ ESBL producers were considered if the isolated pathogens were resistant to one or more extended-spectrum cephalosporins (ceftriaxone, ceftazidime, cefepime), and the double-disk synergy test was applied to identify ESBL producers. ${ }^{14}$ The timeresolved amplified cryptate emission (TRACE) technology assay (Kryptor PCT; Brahms AG; Hennigsdorf, Germany) was adopted to measure serum PCT concentrations. ${ }^{10}$

\section{Study Outcomes}

The primary endpoint of this study was in-hospital mortality. Death episodes during hospitalization that were not related to infection, such as those caused by the underlying malignancy itself or other morbidities, were excluded from the calculations of mortality rate.

\section{Statistical Analysis}

Continuous variables were described as either means and standard deviations or medians and interquartile ranges (IQR) as appropriate. Categorical variables were summarized as counts and percentages. Independent samples $t$-test or Mann-Whitney test were used to compare the continuous variables for data of different groups as appropriate. To compare the categorical variables, chi-square or Fisher's exact tests were used as appropriate means of comparability. The binary logistic regression analysis was adopted to explore the independent prognostic factors for in-hospital mortality of nosocomial infections in GI cancer patients. All variables with a $P$-value $<0.10$ in univariate analysis were further included in multivariate logistic regression analysis using stepwise selection. The tests with a $P$-value $<0.05$ were considered statistically significant. SPSS software version 22.0 for Windows was used to perform all analyses.

\section{Results}

\section{Demographic and Clinical Characteristics} We screened a total of 13,695 cancer patients who admitted to the cancer treatment center from August 2013 to June 2019. In total, 428 eligible GI cancer patients were included in this retrospective study. Among them, a total of $302(70.6 \%)$ patients were male. The median age of all patients was 62.5 years (range, 57-69 years old). There were $224(52.3 \%)$ patients who had a history of tobacco use. Esophageal cancer was the most common diagnosis among the overall population, accounting for $48.4 \%$, followed by rectal cancer $(11.9 \%)$, gastric cancer $(10.0 \%)$, and colon cancer $(9.3 \%)$. A total of 319 (74.5\%) patients had an ECOG performance status lower than two scores. There were 252 (58.9\%) patients who had advanced tumors (stage III-IV), and 105 patients had distant metastases. The most common underlying morbidities were diabetes mellitus identified in 37 (8.6\%) patients, followed by chronic obstructive pulmonary disease (COPD) in 21 (4.9\%) patients, and liver disease in 21 (4.9\%) patients. Among the 428 patients, 248 (58.0\%) had undergone surgery, 103 (24.1\%) had received chemotherapy, $33(7.7 \%)$ had received radiotherapy, and $14(3.3 \%)$ had received concurrent chemoradiotherapy. The majority $(74.3 \%)$ of patients had received invasive procedures within 30 days. A total of $232(54.2 \%)$ patients had drains post-operation, followed by indwelling urinary catheters in $217(50.7 \%)$ cases, nasogastric tube in 181 (42.3\%) cases, and percutaneous pleural drainage tube in $146(34.1 \%)$ cases. The detailed demographic and clinical characteristics of the enrolled patients were summarized in Table 1.

\section{Infection-Related Characteristics}

Of the 428 GI cancer patients with nosocomial infections, a total of $189(44.2 \%)$ patients were confirmed as respiratory tract infections, followed by BSIs in $50(11.7 \%)$ patients, abdominal cavity infections in $49(11.4 \%)$ patients, and thoracic cavity infections in 41 (9.6\%) patients. There were $398(93.0 \%)$ patients had received empirical antibiotic treatment during hospitalization. The most common empirical antibiotic treatment regimens were combination therapy $(52.3 \%)$, followed by $\beta$-lactam 
Table I Demographic and Clinical Characteristics of Nosocomial Infections in Gastrointestinal Cancer Patients

\begin{tabular}{|c|c|c|c|c|c|c|}
\hline \multirow[t]{2}{*}{ Characteristics } & \multirow[t]{2}{*}{ All $N=428$} & \multicolumn{3}{|c|}{ Univariate Analysis } & \multicolumn{2}{|c|}{ Multivariate Analysis } \\
\hline & & $\begin{array}{l}\text { Survivor } \\
N=380, \text { n (\%) }\end{array}$ & $\begin{array}{l}\text { Non-Survivor } \\
N=48, \text { n (\%) }\end{array}$ & P-value & OR $(95 \% \mathrm{Cl})$ & P-value \\
\hline $\begin{array}{l}\text { Demographic data } \\
\text { Sex (male) } \\
\text { Age (years) } \\
\text { Tobacco use }\end{array}$ & $\begin{array}{l}302(70.6) \\
62.5(57.0-69.0) \\
224(52.3)\end{array}$ & $\begin{array}{l}272(71.6) \\
62.0(57.0-69.0) \\
200(52.6)\end{array}$ & $\begin{array}{l}30(62.5) \\
64.0(59.0-72.0) \\
24(50.0)\end{array}$ & $\begin{array}{l}0.193 \\
0.444 \\
0.731\end{array}$ & & \\
\hline $\begin{array}{l}\text { ECOG performance status } \\
0,1 \\
2,3,4\end{array}$ & $\begin{array}{l}319(74.5) \\
109(25.5)\end{array}$ & $\begin{array}{l}303(79.7) \\
77(20.3)\end{array}$ & $\begin{array}{l}16(33.3) \\
32(66.7)\end{array}$ & $<0.001$ & $\begin{array}{l}\text { REF }(1.00) \\
9.99(3.57-27.92)\end{array}$ & $<0.001$ \\
\hline $\begin{array}{l}\text { Underlying cancer type } \\
\text { Esophageal cancer } \\
\text { Gastroesophageal junction } \\
\text { cancer } \\
\text { Gastric cancer } \\
\text { Hepatobiliary cancer } \\
\text { Pancreatic cancer } \\
\text { Colon cancer } \\
\text { Rectal cancer }\end{array}$ & $\begin{array}{l}207(48.4) \\
35(8.2) \\
43(10.0) \\
31(7.2) \\
21(4.9) \\
40(9.3) \\
51(11.9)\end{array}$ & $\begin{array}{l}188(49.5) \\
33(8.7) \\
39(10.3) \\
24(6.3) \\
17(4.5) \\
31(8.2) \\
48(12.6)\end{array}$ & $\begin{array}{l}19(39.6) \\
2(4.2) \\
4(8.3) \\
7(14.6) \\
4(8.3) \\
9(18.8) \\
3(6.3)\end{array}$ & $\begin{array}{l}0.196 \\
0.426 \\
0.869 \\
0.074 \\
0.417 \\
\mathbf{0 . 0 3 5} \\
0.198\end{array}$ & $3.08(0.85-11.17)$ & 0.086 \\
\hline $\begin{array}{l}\text { Existence of distant } \\
\text { metastasis }\end{array}$ & $105(24.5)$ & $82(21.6)$ & $23(47.9)$ & $<0.001$ & $0.44(0.15-1.30)$ & 0.137 \\
\hline $\begin{array}{l}\text { Stage of cancer } \\
\text { I-II } \\
\text { III-IV }\end{array}$ & $\begin{array}{l}176(4 \mid .1) \\
252(58.9)\end{array}$ & $\begin{array}{l}161(42.4) \\
219(57.6)\end{array}$ & $\begin{array}{l}15(31.3) \\
33(68.8)\end{array}$ & 0.140 & & \\
\hline $\begin{array}{l}\text { Comorbidities } \\
\text { COPD } \\
\text { Cerebrovascular disease } \\
\text { Liver disease } \\
\text { Diabetes } \\
\text { Renal disease }\end{array}$ & $\begin{array}{l}21(4.9) \\
8(1.9) \\
21(4.9) \\
37(8.6) \\
12(2.8)\end{array}$ & $\begin{array}{l}13(3.4) \\
5(1.3) \\
13(3.4) \\
31(8.2) \\
9(2.4)\end{array}$ & $\begin{array}{l}8(16.7) \\
3(6.3) \\
8(16.7) \\
6(12.5) \\
3(6.3)\end{array}$ & $\begin{array}{l}<0.001 \\
0.050 \\
<0.001 \\
0.462 \\
0.284\end{array}$ & $\begin{array}{l}4.37(0.4 I-47.17) \\
5.01(0.27-94.27) \\
3.01(0.37-24.33)\end{array}$ & $\begin{array}{l}0.225 \\
0.282 \\
0.301\end{array}$ \\
\hline $\begin{array}{l}\text { CCI } \\
0 \\
\mathrm{I}-2 \\
\geq 3\end{array}$ & $\begin{array}{l}344(80.4) \\
75(17.5) \\
9(2.1)\end{array}$ & $\begin{array}{l}316(83.2) \\
60(15.8) \\
4(1.1)\end{array}$ & $\begin{array}{l}28(58.3) \\
15(31.3) \\
5(10.4)\end{array}$ & $<0.001$ & $\begin{array}{l}\text { REF (I.00) } \\
0.51(0.11-2.39) \\
0.96(0.05-18.22)\end{array}$ & $\begin{array}{l}0.638 \\
0.391 \\
0.976\end{array}$ \\
\hline Existence of fever & $188(43.9)$ & $163(42.9)$ & $25(52.1)$ & 0.227 & & \\
\hline $\begin{array}{l}\text { Surgery (within } 30 \text { days) } \\
\text { None } \\
\text { Curative surgery } \\
\text { Palliative surgery }\end{array}$ & $\begin{array}{l}180(42.1) \\
225(52.6) \\
23(5.4)\end{array}$ & $\begin{array}{l}139(36.6) \\
221(58.2) \\
20(5.3)\end{array}$ & $\begin{array}{l}4 I(85.4) \\
4(8.3) \\
3(6.3)\end{array}$ & $<0.001$ & $\begin{array}{l}\text { REF }(1.00) \\
0.09(0.01-1.19) \\
0.20(0.02-1.69)\end{array}$ & $\begin{array}{l}0.168 \\
0.067 \\
0.140\end{array}$ \\
\hline $\begin{array}{l}\text { Chemotherapy (within } \\
30 \text { days) } \\
\text { None } \\
\text { Neoadjuvant } \\
\text { Adjuvant } \\
\text { Ist line } \\
\text { 2nd line } \\
\geq 3 \text { rd line }\end{array}$ & $\begin{array}{l}325(75.9) \\
8(1.9) \\
43(10.0) \\
40(9.3) \\
7(1.6) \\
5(1.2)\end{array}$ & $\begin{array}{l}285(75.0) \\
7(1.8) \\
40(10.5) \\
38(10.0) \\
6(1.6) \\
4(1.1)\end{array}$ & $\begin{array}{l}40(83.3) \\
I(2.1) \\
3(6.3) \\
2(4.2) \\
1(2.1) \\
I(2.1)\end{array}$ & 0.613 & & \\
\hline
\end{tabular}


Table I (Continued).

\begin{tabular}{|c|c|c|c|c|c|c|}
\hline \multirow[t]{2}{*}{ Characteristics } & \multirow[t]{2}{*}{ All $N=428$} & \multicolumn{3}{|c|}{ Univariate Analysis } & \multicolumn{2}{|c|}{ Multivariate Analysis } \\
\hline & & $\begin{array}{l}\text { Survivor } \\
\mathbf{N}=380, \text { n (\%) }\end{array}$ & $\begin{array}{l}\text { Non-Survivor } \\
N=48, \text { n (\%) }\end{array}$ & P-value & OR $(95 \% \mathrm{Cl})$ & P-value \\
\hline Radiotherapy (within 30 days) & $33(7.7)$ & $23(6.1)$ & $10(20.8)$ & 0.001 & $2.66(0.53-13.32)$ & 0.234 \\
\hline $\begin{array}{l}\text { Concurrent } \\
\text { chemoradiotherapy } \\
\text { (within } 30 \text { days) }\end{array}$ & $14(3.3)$ & $13(3.4)$ & $I(2.1)$ & 0.952 & & \\
\hline $\begin{array}{l}\text { Corticosteroid therapy } \\
\text { (within } 30 \text { days) }\end{array}$ & $128(29.9)$ & III (29.2) & $17(35.4)$ & 0.376 & & \\
\hline $\begin{array}{l}\text { Prior G-CSF use (within } \\
30 \text { days) }\end{array}$ & $79(18.5)$ & $65(17.1)$ & $14(29.2)$ & 0.042 & $0.30(0.07-1.28)$ & 0.104 \\
\hline $\begin{array}{l}\text { Invasive procedure (within } \\
30 \text { days) }\end{array}$ & $318(74.3)$ & $292(76.8)$ & $26(54.2)$ & 0.001 & $0.77(0.26-2.3 \mathrm{I})$ & 0.645 \\
\hline \multicolumn{7}{|l|}{$\begin{array}{l}\text { Presence of indwelling } \\
\text { catheters } \\
\text { or other devices }\end{array}$} \\
\hline Ureteral stent & $15(3.5)$ & $10(2.6)$ & $5(10.4)$ & 0.019 & $0.94(0.13-6.85)$ & 0.949 \\
\hline Indwelling urinary catheters & $217(50.7)$ & $212(55.8)$ & $5(10.4)$ & $<0.001$ & $0.31(0.02-4.28)$ & 0.380 \\
\hline CVC (port-a-cath or PICC) & $56(13.1)$ & $49(12.9)$ & $7(14.6)$ & 0.744 & & \\
\hline $\begin{array}{l}\text { Percutaneous pleural drainage } \\
\text { tube }\end{array}$ & $146(34.1)$ & I4I (37.I) & $5(10.4)$ & $<0.001$ & $\mathrm{I} .0 \mathrm{I}(0.23-4.50)$ & 0.988 \\
\hline $\begin{array}{l}\text { Percutaneous abdomen } \\
\text { drainage tube }\end{array}$ & $19(4.4)$ & $13(3.4)$ & $6(12.5)$ & 0.012 & $2.01(0.39-10.28)$ & 0.403 \\
\hline Drains post-operation & $232(54.2)$ & $224(58.9)$ & $8(16.7)$ & $<0.001$ & $1.33(0.16-11.34)$ & 0.794 \\
\hline Nasogastric tube & $|8|(42.3)$ & $175(46.1)$ & $6(12.5)$ & $<0.001$ & $2.39(0.29-19.65)$ & 0.416 \\
\hline
\end{tabular}

Note: Bold values indicate statistical significance.

Abbreviations: OR, odds ratio; $\mathrm{Cl}$, confidence interval; ECOG, Eastern Cooperative Oncology Group; COPD, chronic obstructive pulmonary disease, CCI, Charlson comorbidity index score; G-CSF, granulocyte colony-stimulating factor; CVC, central venous catheter; PICC, peripherally inserted central catheter.

$/ \beta$-lactamase inhibitor combinations $(15.9 \%)$, and cephalosporins (11.0\%). A total of 239 (55.8\%) patients were confirmed as to have had received effective empirical antibiotic treatment. The median length of antibiotic treatment for all patients was 9.0 days (range, 6.0-13.0 days). During hospitalization, $73(17.1 \%)$ patients were treated in the ICU, 44 (10.3\%) received mechanical ventilation, and $49(11.4 \%)$ presented septic shock. Finally, a total of 48 $(11.2 \%)$ patients died due to nosocomial infections. The median length of hospitalization of all patients was 21.0 days (range, 11.0-28.0 days). The detailed infectionrelated characteristics of the included patients were summarized in Table 2.

\section{Microbiology Characteristics}

A total of 428 strains of causative pathogens were isolated from different clinical samples during the study period. The most common samples were sputum (40.9\%), followed by blood $(14.5 \%)$, drainage fluid $(13.1 \%)$, and urine $(8.4 \%)$. Gram-negative bacteria (GNB) were the leading causative pathogens of nosocomial infections in GI cancer patients, accounting for $36.7 \%$, followed by MDRGNB (27.6\%), polymicrobial (13.6\%), Grampositive bacteria (9.6\%), and fungi $(9.6 \%)$. Of the isolated $\mathrm{GNB}$, the most frequently isolated pathogens were $E$. coli (11.9\%), Klebsiella pneumoniae (10.0\%), and Pseudomonas aeruginosa (9.1\%). Among the MDRGNB group, ESBL-producing E. coli (13.6\%), Acinetobacter baumannii (4.7\%), and ESBL-producing Klebsiella pneumoniae $(3.7 \%)$ were the most frequently isolated pathogens. The isolated Gram-positive bacteria mainly included Staphylococcus aureus (3.7\%), Streptococcus pneumoniae $(2.8 \%)$, and Streptococcus anginosus (1.6\%). The detailed microbiological characteristics were presented in Table 3.

\section{Antimicrobial Susceptibility Analysis}

We then performed antimicrobial susceptibility analysis of the isolated causative pathogens. It showed that the 
Table 2 Infection-Related Characteristics of Nosocomial Infections in Gastrointestinal Cancer Patients

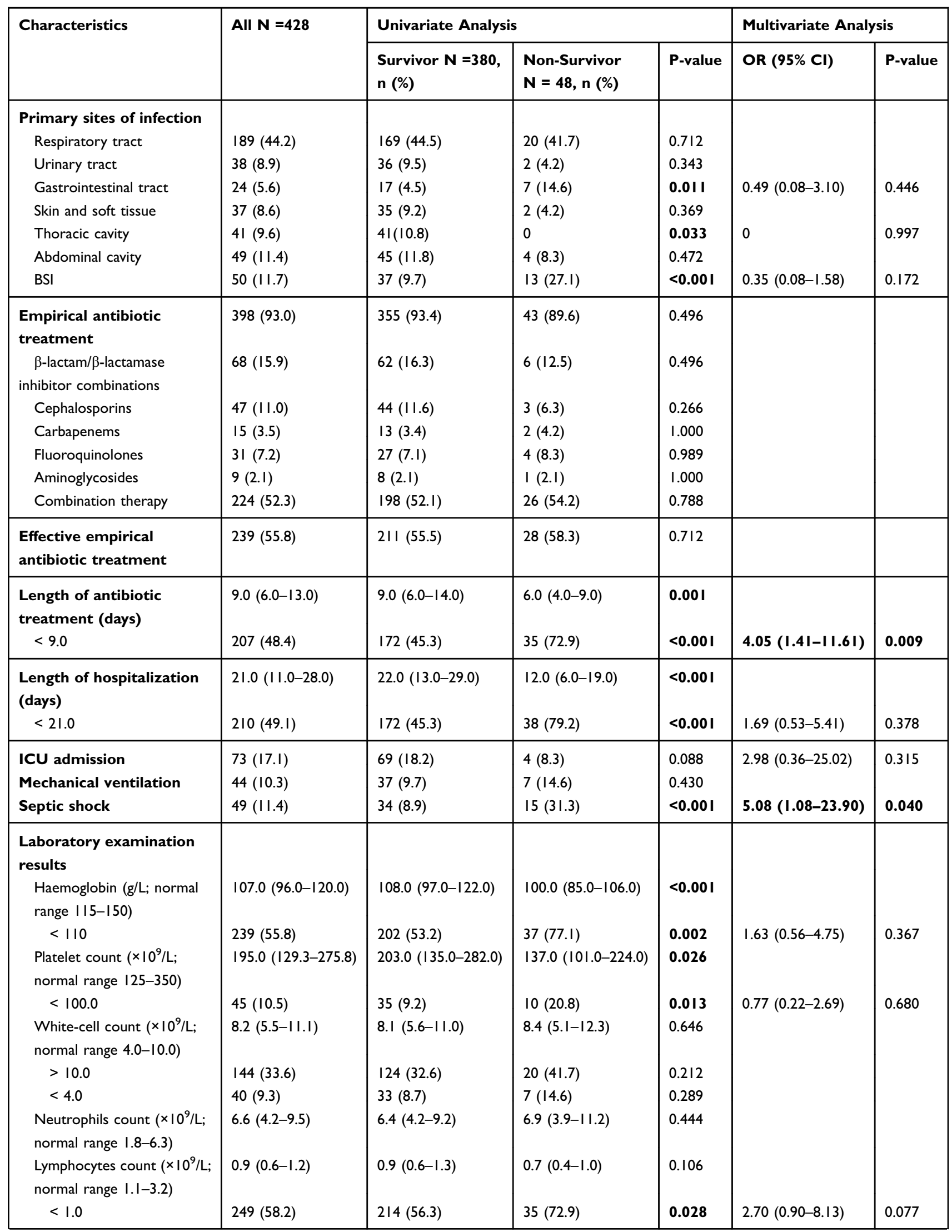


Table 2 (Continued).

\begin{tabular}{|c|c|c|c|c|c|c|}
\hline \multirow[t]{2}{*}{ Characteristics } & \multirow[t]{2}{*}{ All $N=428$} & \multicolumn{3}{|l|}{ Univariate Analysis } & \multicolumn{2}{|c|}{ Multivariate Analysis } \\
\hline & & $\begin{array}{l}\text { Survivor N =380, } \\
\text { n (\%) }\end{array}$ & $\begin{array}{l}\text { Non-Survivor } \\
N=48, n(\%)\end{array}$ & P-value & OR $(95 \% \mathrm{Cl})$ & P-value \\
\hline $\begin{array}{l}\mathrm{PCT}^{\mathrm{a}}(\mathrm{ng} / \mathrm{mL} ; \text { normal range } \\
0-0.5)\end{array}$ & $0.5(0.4-2.1)$ & $0.5(0.3-1.9)$ & $0.5(0.4-3.7)$ & 0.906 & & \\
\hline$\geq 1.0$ & $96(33.4)$ & $84(33.2)$ & $12(35.3)$ & 0.808 & & \\
\hline $\begin{array}{l}\text { Albumin (g/L; normal range } \\
40-55)\end{array}$ & $32.0(29.0-36.0)$ & $33.0(29.0-36.0)$ & $30.0(27.0-33.0)$ & 0.002 & & \\
\hline$<30.0$ & 145 (33.9) & $119(31.3)$ & $26(54.2)$ & 0.002 & $4.50(1.58-\mid 2.83)$ & 0.005 \\
\hline
\end{tabular}

Notes: a Only 287 patients' data are available. Bold values indicate statistical significance.

Abbreviations: $\mathrm{OR}$, odds ratio; $\mathrm{Cl}$, confidence interval; $\mathrm{BSI}$, bloodstream infection; ICU, intensive care unit; PCT, procalcitonin.

isolated GNB exhibited high sensitivity to amikacin (94.5\%), meropenem (92.4\%), imipenem (91.2\%), piperacillin/tazobactam (83.4\%), and cefepime (78.0\%), while they were mainly resistant to ceftriaxone $(48.7 \%)$, sulfamethoxazole-trimethoprim (45.4\%), aztreonam (39.1\%), and ciprofloxacin (29.2\%) (Figure 1A). Compared with the isolated GNB, the isolated Gram-positive bacteria exhibited high sensitivity to tigecycline $(100.0 \%)$, linezolid $(100.0 \%)$, minocycline $(100.0 \%)$, and vancomycin $(98.0 \%)$, while they were mainly resistant to clindamycin $(75.0 \%)$, penicillin $(51.1 \%)$, tetracycline $(45.2 \%)$, and sulfamethoxazole-trimethoprim (44.4\%) (Figure 1B).

\section{Prognostic Factors for In-Hospital Mortality of Gastrointestinal Cancer Patients with Nosocomial Infections}

A total of 48 (11.2\%) patients died of nosocomial infections during hospitalization. The results of the univariate analysis identified several variables were statistically significant for in-hospital mortality (Tables 1-3). The results of the multivariate analysis revealed that ECOG performance status ztwo scores (OR 9.99; 95\% CI 3.57-27.92), length of antibiotic treatment $<9.0$ days (OR $4.05 ; 95 \%$ CI $1.41-11.61$ ), existence of septic shock (OR 5.08; 95\% CI 1.08-23.90), and hypoproteinemia (OR 4.50; 95\% CI 1.58-12.83) were independent risk factors for in-hospital mortality of hospitalized GI cancer patients with nosocomial infections.

\section{Discussion}

GI cancer patients represent a particular group of people since these patients are predisposed to developing immunosuppression due to a variety of different mechanisms. ${ }^{15,16}$ As a result, infections remain one of the most common complications for these patients during hospitalization. ${ }^{17}$ Besides, nosocomial infections in cancer patients are associated with prolonged hospitalization, delayed initiation of chemotherapy, increased medical costs, and substantial morbidity and mortality. Therefore, we performed this study to describe the bacteria distribution, antibiotic resistance pattern, and clinical outcomes of nosocomial infections in hospitalized GI cancer patients.

The global epidemiology of nosocomial infections in cancer patients has changed over time. ${ }^{2}$ Throughout the 1960s and 1970s, GNB were the most common causative pathogens in cancer patients. During the next 20 years, the causative pathogens shifted from GNB to Gram-positive bacteria due to chemotherapy-induced severe oral mucositis, the widespread use of central venous catheters, and antibacterial prophylaxis administration. ${ }^{18}$ However, in recent years, a new re-emergence of GNB as leading causative pathogens of infections were reported worldwide. ${ }^{19-21}$ In consistent, our results showed that $36.7 \%$ of cases were caused by GNB, and 9.6\% of cases were caused by Gram-positive bacteria. Similar to previously published studies, ${ }^{6,15,22,23}$ we found that E. coli, Klebsiella pneumoniae, and Pseudomonas aeruginosa were the most frequently isolated causative pathogens of nosocomial infections caused by GNB. Among the isolated Gram-positive bacteria, Staphylococcus aureus was the most commonly isolated causative pathogen, followed by Streptococcus pneumoniae. Similarly, Antonio et al reported that Staphylococcus aureus and Streptococcus pneumoniae were the most frequently isolated causative pathogens of nosocomial infections caused by Gram-positive bacteria among elderly cancer patients in Spain. ${ }^{22}$ Nosocomial infections due to fungi were observed in $9.6 \%$ of cases. Among these, Candida albicans was the most commonly isolated causative pathogen. This is consistent with the previous study conducted by Calik Basaran et al in a tertiary care 
Table 3 Causative Pathogens of All Nosocomial Infection Episodes in Gastrointestinal Cancer Patients

\begin{tabular}{|c|c|c|c|c|c|c|}
\hline \multirow[t]{2}{*}{ Causative Organisms } & \multirow{2}{*}{$\begin{array}{l}\text { All } \\
N=428\end{array}$} & \multicolumn{3}{|c|}{ Univariate Analysis } & \multicolumn{2}{|c|}{ Multivariate Analysis } \\
\hline & & $\begin{array}{l}\text { Survivor } \mathbf{N}=\mathbf{3 8 0} \text {, } \\
\text { n (\%) }\end{array}$ & $\begin{array}{l}\text { Non-Survivor } \mathbf{N}=\mathbf{4 8} \\
n \text { (\%) }\end{array}$ & P-value & OR $(95 \% \mathrm{CI})$ & P-value \\
\hline Gram-negative bacteria & $157(36.7)$ & $146(38.4)$ & II (22.9) & 0.036 & & \\
\hline Escherichia coli & $51(11.9)$ & $50(13.2)$ & $\mathrm{I}(2.1)$ & 0.026 & $0.06(0.00-1.34)$ & 0.075 \\
\hline Klebsiella pneumoniae & $43(10.0)$ & 42 (II.I) & I (2.I) & 0.090 & $0.23(0.02-2.20)$ & 0.202 \\
\hline Pseudomonas aeruginosa & $39(9.1)$ & $36(9.5)$ & $3(6.3)$ & 0.642 & & \\
\hline Enterobacter spp. & $24(5.6)$ & $18(4.7)$ & $6(12.5)$ & 0.061 & $3.29(0.76-14.23)$ & 0.112 \\
\hline Gram-positive bacteria & $4 I(9.6)$ & $38(10.0)$ & $3(6.3)$ & 0.568 & & \\
\hline Staphylococcus aureus & $16(3.7)$ & $13(3.4)$ & $3(6.3)$ & 0.569 & & \\
\hline Streptococcus pneumoniae & $12(2.8)$ & $12(3.2)$ & 0 & 0.433 & & \\
\hline Coagulase-negative & $6(1.4)$ & $6(1.6)$ & 0 & 1.000 & & \\
\hline \multicolumn{7}{|l|}{ Staphylococci } \\
\hline Streptococcus anginosus & $7(1.6)$ & $7(1.8)$ & 0 & 1.000 & & \\
\hline Fungi & $4 \mid(9.6)$ & $31(8.2)$ & $10(20.8)$ & 0.011 & & \\
\hline Candida albicans & $31(7.2)$ & $24(6.3)$ & $7(14.6)$ & 0.074 & $3.96(0.93-16.91)$ & 0.063 \\
\hline Candida spp. & $5(1.2)$ & $3(0.8)$ & $2(4.2)$ & 0.099 & $6.18(0.21-185.43)$ & 0.294 \\
\hline Eurotium & $5(1.2)$ & $4(1.1)$ & $\mathrm{I}(2.1)$ & 0.450 & & \\
\hline MDRGNB & $118(27.6)$ & $103(27.1)$ & $15(3 \mid .3)$ & 0.545 & & \\
\hline ESBL-producing Escherichia coli & $58(13.6)$ & $47(12.4)$ & II (22.9) & 0.124 & & \\
\hline ESBL-producing Klebsiella & $16(3.7)$ & $16(4.2)$ & 0 & 0.274 & & \\
\hline pneumoniae & & & & & & \\
\hline MDR Pseudomonas aeruginosa & $7(1.6)$ & $7(1.8)$ & 0 & 1.000 & & \\
\hline Acinetobacter baumannii & $20(4.7)$ & $18(4.7)$ & $2(4.2)$ & 1.000 & & \\
\hline Stenotrophomonas maltophilia & $14(3.3)$ & $12(3.2)$ & $2(4.2)$ & 1.000 & & \\
\hline CRE & $3(0.7)$ & $3(0.8)$ & 0 & 1.000 & & \\
\hline Enterococcus & $10(2.3)$ & $6(1.6)$ & $4(8.3)$ & 0.016 & $3.47(0.42-28.94)$ & 0.250 \\
\hline Anaerobes & $3(0.7)$ & $3(0.8)$ & 0 & 1.000 & & \\
\hline Polymicrobial & $58(13.6)$ & $53(13.9)$ & $5(10.4)$ & 0.501 & & \\
\hline
\end{tabular}

Note: Bold values indicate statistical significance.

Abbreviations: MDRGNB, multidrug-resistant gram-negative bacteria; ESBL, extended-spectrum $\beta$-lactamase; MDR, multidrug-resistant; CRE, carbapenem-resistant Enterobacteriaceae.

academic center in Turkey. ${ }^{24}$ In the present study, it is noteworthy that MDR strains were detected in $27.6 \%$ of infection episodes. ESBL-producing E. coli and Acinetobacter baumannii were the most frequently isolated causative pathogens of nosocomial infections caused by MDRGNB. Besides, there were no MDR Gram-positive bacteria detected in our study. Previous studies reported that ESBL-producing Enterobacteriaceae was the leading causative pathogen for nosocomial infections in cancer patients caused by MDR strains. ${ }^{13,15,25}$

Immunosuppressed cancer patients are prone to developing septic shock due to severe infections during hospitalization. Therefore, appropriate and adequate empirical antibiotic treatment plays an important role in reducing infection-related mortality. ${ }^{2}$ However, previously published guidelines only addressed the antimicrobial therapy of nosocomial infections in cancer patients with febrile neutropenia $(\mathrm{FN}){ }^{26-28}$ Gudiol et al reported that $\beta$-lactam $+\beta$-lactamase inhibitor combination was the most frequent empirical antimicrobial regimen in dealing with infections among cancer patients, followed by oxymino- $\beta$-lactams. ${ }^{2}$ In our study, the results of antimicrobial susceptibility analysis demonstrated that the isolated GNB exhibited high sensitivity to amikacin, meropenem, imipenem, and piperacillin/tazobactam. However, the isolated GNB exhibited high resistance to ceftriaxone, sulfamethoxazole-trimethoprim, and aztreonam. Ashour et al also reported a comparable observation among hospitalized cancer patients in Egypt. ${ }^{6}$ It is reported that the colonization of ESBL-producers has been increased dramatically in recent years. ${ }^{2}$ In the present study, ESBL-producing 

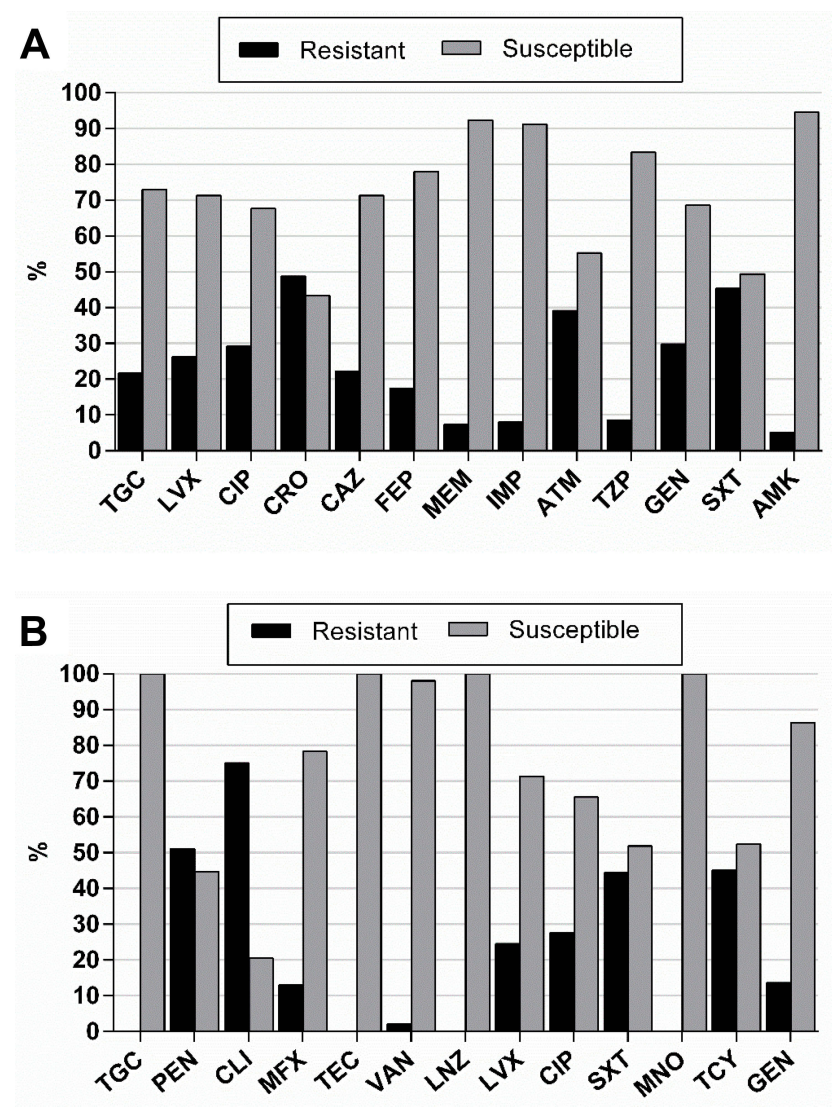

Figure I Antimicrobial susceptibility comparison among gastrointestinal cancer patients with nosocomial infections. (A) The isolated Gram-negative bacteria. (B) The isolated Gram-positive bacteria.

Abbreviations: TGC, tigecycline; LVX, levofloxacin; CIP, ciprofloxacin; CRO, ceftriaxone; CAZ, ceftazidime; FEP, cefepime; MEM, meropenem; IMP, imipenem; ATM, aztreonam; TZP, piperacillin/tazobactam; GEN, gentamicin; SXT, sulfamethoxazole-trimethoprim; AMK, amikacin; PEN, penicillin; CLI, clindamycin; MFX, moxifloxacin; TEC, teicoplanin; VAN, vancomycin; LNZ, linezolid; MNO, minocycline; TCY, tetracycline.

Enterobacteriaceae was the most commonly isolated MDR strains. Ceftriaxone and aztreonam resistance are potential markers for the presence of ESBL. The isolated Grampositive bacteria exhibited high sensitivity to tigecycline, linezolid, and vancomycin since there were no MDR strains detected in this group. A previous study reported a meager resistance rate to these antibiotics in non-neutropenic cancer patients in Taiwan, China. ${ }^{29}$

The overall in-hospital mortality of all patients was $11.2 \%(48 / 428)$ in the study. This finding was lower as compared with the previous retrospective studies performed in other centers (17.0-32.0\%). ${ }^{15,22-24}$ It may be attributed to the fact that only a small number of patients developed BSIs during hospitalization in our study. Besides, there were few patients who developed neutropenia due to the appropriate administration of G-CSF. The results of multivariate analysis revealed that ECOG performance status, length of antibiotic treatment, septic shock, and serum albumin level were associated with the in-hospital mortality of GI cancer patients with nosocomial infections. Cancer patients with higher ECOG performance status were associated with worse prognoses. We found that ECOG performance status $\geq$ two scores was significantly correlated with increased inhospital mortality rate in our study. It suggests that we should focus more on these patients once they were confirmed as infection episodes during hospitalization. Length of antibiotic treatment $<9.0$ days and septic shock were also identified as risk factors associated with unfavorable clinical outcomes, which is aligned with the previous studies conducted in Spain ${ }^{22,30}$ and Taiwan, China. ${ }^{31}$ In addition, we also found that hypoproteinemia was an independent risk factor of in-hospital mortality. Similarly observation was made in a retrospective study conducted in China. ${ }^{32}$ Previous studies revealed that low serum albumin levels were associated with poor nutrition status and weight loss in cancer patients, and hypoproteinemia was positively associated with short - and long-term mortality in all hospitalized patients. ${ }^{32,33}$

Our study also has several limitations. First, this is a retrospective study. Although partial infection episodes were determined under the clinical manifestations of patients by qualified physicians, there might be potential bias in the study. Furthermore, the chemotherapy regimens, radiotherapy dosage, prior infection history, and prior antibiotic treatment were not collected. Moreover, considering this retrospective study was carried out in one hospital, further prospective multicenter studies are warranted to verify our results.

\section{Conclusions}

The burden of nosocomial infections in GI cancer patients is considerably high, with GNB being predominantly isolated causative pathogens. MDR strains were detected in $27.6 \%$ of isolates. The isolated GNB exhibited high sensitivity to amikacin, meropenem, imipenem, and piperacillin/tazobactam, while the isolated Gram-positive bacteria exhibited high sensitivity to tigecycline, linezolid, and vancomycin. Our findings also suggest that ECOG performance status $\geq$ two scores, inadequate antibiotic treatment, septic shock, and hypoproteinemia were associated with increased in-hospital mortality. Surveillance on serum albumin level, adequate antibiotic treatment, early identification and prompt treatment of septic shock could advance the prognosis of nosocomial infections in GI cancer patients. 


\section{Ethics Approval and Consent to Participate}

This study was approved by the ethics committee of the First Affiliated Hospital of Xi' an Jiaotong University (No: XJTU1AF2020LSK-049). Waiving of informed consent was obtained due to the retrospective noninterventional study design. Furthermore, we confirm that the patient data accessed complied with data protection privacy laws.

\section{Author Contributions}

All authors made substantial contributions to conception and design, acquisition of data, or analysis and interpretation of data; took part in drafting the article or revising it critically for important intellectual content; gave final approval of the version to be published; and agree to be accountable for all aspects of the work.

\section{Funding}

This research received no specific grant from any funding agency in the public, commercial, or not-for-profit sectors.

\section{Disclosure}

No potential conflict of interest was reported by the authors.

\section{References}

1. Global Burden of Disease Cancer C, Fitzmaurice C, Allen C, et al. Global, regional, and national cancer incidence, mortality, years of life lost, years lived with disability, and disability-adjusted life-years for 32 cancer groups, 1990 to 2015: a systematic analysis for the global burden of disease study. JAMA Oncol. 2017;3(4):524-548. doi:10.10 01/jamaoncol.2016.5688

2. Gudiol C, Aguado JM, Carratala J. Bloodstream infections in patients with solid tumors. Virulence. 2016;7(3):298-308. doi:10.1080/21505 594.2016.1141161

3. Gajdacs M, Urban E. Epidemiological trends and resistance associated with stenotrophomonas maltophilia bacteremia: a 10-year retrospective cohort study in a tertiary-care hospital in Hungary. Diseases. 2019;7:2. doi:10.3390/diseases 7020041

4. Brand JS, Colzani E, Johansson ALV, et al. Infection-related hospitalizations in breast cancer patients: risk and impact on prognosis. $J$ Infect. 2016;72(6):650-658. doi:10.1016/j.jinf.2016.04.003

5. Kamboj M, Sepkowitz KA. Nosocomial infections in patients with cancer. Lancet Oncol. 2009;10(6):589-597. doi:10.1016/S1470-2045 (09)70069-5

6. Ashour HM, El-Sharif A. Species distribution and antimicrobial susceptibility of gram-negative aerobic bacteria in hospitalized cancer patients. J Transl Med. 2009;7:14. doi:10.1186/1479-5876-7-14

7. Charlson ME, Pompei P, Ales KL, MacKenzie CR. A new method of classifying prognostic comorbidity in longitudinal studies: development and validation. J Chronic Dis. 1987;40(5):373-383. doi:10.10 16/0021-9681(87)90171-8

8. Crowe MJ, Cooke EM. Review of case definitions for nosocomial infection - towards a consensus. J Hosp Infect. 1998;39(1):3-11. doi:10.1016/S0195-6701(98)90237-7
9. Perdikouri EIA, Arvaniti K, Lathyris D, et al. Infections due to multidrug-resistant bacteria in oncological patients: insights from a five-year epidemiological and clinical analysis. Microorganisms. 2019;7:9. doi:10.3390/microorganisms7090277

10. Vincenzi B, Fioroni I, Pantano F, et al. Procalcitonin as diagnostic marker of infection in solid tumors patients with fever. Sci Rep. 2016;6:28090. doi:10.1038/srep28090

11. Shankar-Hari M, Phillips GS, Levy ML, et al. Developing a new definition and assessing new clinical criteria for septic shock: for the third international consensus definitions for sepsis and septic shock (sepsis-3). JAMA. 2016;315(8):775-787. doi:10.1001/jama.2016.0289

12. Gajdacs M, Batori Z, Abrok M, Lazar A, Burian K. Characterization of resistance in gram-negative urinary isolates using existing and novel indicators of clinical relevance: a 10-year data analysis. Life (Basel). 2020;10(2):16.

13. CLSI. Performance Standards for Antimicrobial Susceptibility Testing, Supplement M100S. 26th ed. Wayne, PA, USA: Clinical and Laboratory Standards Institute; 2016.

14. Ben-David D, Kordevani R, Keller N, et al. Outcome of carbapenem resistant klebsiella pneumoniae bloodstream infections. Clin Microbiol Infect. 2012;18(1):54-60. doi:10.1111/j.1469-0691.2011. 03478.x

15. Marin M, Gudiol C, Garcia-Vidal C, Ardanuy C, Carratala J. Bloodstream infections in patients with solid tumors: epidemiology, antibiotic therapy, and outcomes in 528 episodes in a single cancer center. Medicine (Baltimore). 2014;93(3):143-149. doi:10.1097/ MD.0000000000000026

16. Safdar A, Armstrong D. Infectious morbidity in critically ill patients with cancer. Crit Care Clin. 2001;17(3):531-570. doi:10.1016/ S0749-0704(05)70198-6

17. Fentie A, Wondimeneh Y, Balcha A, Amsalu A, Adankie BT. Bacterial profile, antibiotic resistance pattern and associated factors among cancer patients at University of Gondar Hospital, Northwest Ethiopia. Infect Drug Resist. 2018;11:2169-2178. doi:10.2147/IDR. $\mathrm{S} 183283$

18. Cordonnier C, Buzyn A, Leverger G, et al. Epidemiology and risk factors for gram-positive coccal infections in neutropenia: toward a more targeted antibiotic strategy. Clin Infect Dis. 2003;36 (2):149-158. doi:10.1086/345435

19. Gajdacs M. The concept of an ideal antibiotic: implications for drug design. Molecules. 2019;24(5):892. doi:10.3390/molecules24050892

20. Wisplinghoff H, Seifert H, Wenzel RP, Edmond MB. Current trends in the epidemiology of nosocomial bloodstream infections in patients with hematological malignancies and solid neoplasms in hospitals in the United States. Clin Infect Dis. 2003;36(9):1103-1110. doi:10. $1086 / 374339$

21. Gudiol C, Bodro M, Simonetti A, et al. Changing aetiology, clinical features, antimicrobial resistance, and outcomes of bloodstream infection in neutropenic cancer patients. Clin Microbiol Infect. 2013;19(5):474-479. doi:10.1111/j.1469-0691.2012.03879.x

22. Antonio M, Gudiol C, Royo-Cebrecos C, Grillo S, Ardanuy C, Carratala J. Current etiology, clinical features and outcomes of bacteremia in older patients with solid tumors. J Geriatr Oncol. 2019;10 (2):246-251. doi:10.1016/j.jgo.2018.06.011

23. Aydemir H, Piskin N, Kokturk F, Gokmen A, Akduman D. Healthcare associated bacteremia in geriatric cancer patients with febrile neutropenia. J Geriatr Oncol. 2013;4(2):190-195. doi:10.1016/j. jgo.2012.10.174

24. Calik Basaran N, Karaagaoglu E, Hascelik G, Durusu Tanriover M, Akova M. Prospective evaluation of infection episodes in cancer patients in a tertiary care academic center: microbiological features and risk factors for mortality. Turk J Haematol. 2016;33(4):311-319. doi:10.4274/tjh.2015.0216

25. Ariza-Heredia EJ, Chemaly RF. Update on infection control practices in cancer hospitals. CA Cancer J Clin. 2018;68(5):340-355. doi: $10.3322 /$ caac. 21462 
26. Freifeld AG, Bow EJ, Sepkowitz KA, et al. Clinical practice guideline for the use of antimicrobial agents in neutropenic patients with cancer: 2010 update by the infectious diseases society of America. Clin Infect Dis. 2011;52(4):427-431. doi:10.1093/cid/ciq147

27. Kochanek M, Schalk E, von Bergwelt-baildon M, et al. Management of sepsis in neutropenic cancer patients: 2018 guidelines from the Infectious Diseases Working Party (AGIHO) and Intensive Care Working Party (iCHOP) of the German Society of Hematology and Medical Oncology (DGHO). Ann Hematol. 2019;98(5):1051-1069. doi:10.1007/s00277-019-03622-0

28. Heinz WJ, Buchheidt D, Christopeit M, et al. Diagnosis and empirical treatment of fever of unknown origin (FUO) in adult neutropenic patients: guidelines of the Infectious Diseases Working Party (AGIHO) of the German Society of Hematology and Medical Oncology (DGHO). Ann Hematol. 2017;96(11):1775-1792. doi:10.1007/s00277-017-3098-3

29. Huang CC, Wu CJ, Wang LR, et al. Antimicrobial susceptibility of bacteremic isolates from cancer patients with or without neutropenia at a medical center in southern Taiwan. J Microbiol Immunol Infect. 2011;44(5):376-381. doi:10.1016/j.jmii.2011.01.035
30. Palacios-Baena ZR, Gutierrez-Gutierrez B, De Cueto M, et al. Development and validation of the INCREMENT-ESBL predictive score for mortality in patients with bloodstream infections due to extended-spectrum-beta-lactamase-producing enterobacteriaceae. J Antimicrob Chemother. 2017;72(3):906-913. doi:10.1093/jac/ dkw513

31. Chen CY, Tien FM, Sheng WH, et al. Clinical and microbiological characteristics of bloodstream infections among patients with haematological malignancies with and without neutropenia at a medical centre in northern Taiwan, 2008-2013. Int J Antimicrob Agents. 2017;49(3):272-281. doi:10.1016/j.ijantimicag.2016.11.009

32. Xiao T, Yang K, Zhou Y, et al. Risk factors and outcomes in non-transplant patients with extended-spectrum beta-lactamaseproducing Escherichia coli bacteremia: a retrospective study from 2013 to 2016. Antimicrob Resist Infect Control. 2019;8:144. doi:10.1186/s13756-019-0599-y

33. Paccagnella A, Morassutti I, Rosti G. Nutritional intervention for improving treatment tolerance in cancer patients. Curr Opin Oncol. 2011;23(4):322-330. doi:10.1097/CCO.0b013e3283479c66

\section{Publish your work in this journal}

Cancer Management and Research is an international, peer-reviewed open access journal focusing on cancer research and the optimal use of preventative and integrated treatment interventions to achieve improved outcomes, enhanced survival and quality of life for the cancer patient.
The manuscript management system is completely online and includes a very quick and fair peer-review system, which is all easy to use. Visit http://www.dovepress.com/testimonials.php to read real quotes from published authors. 